\title{
EVALUATION OF EFFICIENCY AND SAFETY OF PIEZOSURGERY VERSUS CONVENTIONAL ROTARY INSTRUMENTS IN LATERAL MAXILLARY SINUS FLOOR ELEVATION: A PROSPECTIVE RANDOMIZED CONTROLLED CLINICAL TRIAL
}

\author{
Mohamed Shokry", Rehab Tarek Elsharkawy ${ }^{* *}$ and Hani Arakji***
}

\begin{abstract}
To overcome the intra and post-operative complications that might arise during the use of the conventional instruments for cutting the bony window and elevation of maxillary sinus membrane, the application of ultrasonic device (piezosurgery) has been introduced.
\end{abstract}

Aim of the study: To evaluate the efficiency and safety of the piezosurgery during direct sinus lifting procedure in comparison to the conventional rotary instruments.

Patients and methods: A randomized controlled clinical trial was performed, in which forty patients (17 females and 23 males) with bone height of less than $3 \mathrm{~mm}$ in the posterior maxilla, who required implant placement to replace the missing teeth, were selected. The sinus lift surgeries were done either by the piezosurgery or the conventional bur technique. The rate of perforation, time of the operation, healing, postoperative edema, and pain were evaluated. Six months later, implants were placed within the augmented sites.

Results: A lower percentage of membrane perforation has been encountered in the piezosurgery group ( $5 \%$ one patient), when compared to the conventional group (15\%, three patients).The pain and swelling were significantly less in the piezosurgery group but the mean time was longer in the piezosurgery group .

Conclusion: The piezosurgery is an effective device that is safer in performing the lateral maxillary sinus lift surgery than the conventional rotary method, but unfortunately it takes longer operation time.

KEYWORDS: sinus lift, schneiderian membrane, piezosurgery, rotary instruments, dental implants.

\footnotetext{
*Assistant Professor of Oral and Maxillofacial Surgery, Faculty of Dentistry, Alexandria University. **Assistant Professor of Oral and Maxillofacial Surgery, Faculty of Dentistry, Cairo University. *** Oral Surgeon and Implantologist, Private practice.
} 


\section{INTRODUCTION}

Pneumatization of the maxillary sinus, with subsequent reduction of the alveolar bone height, usually occurs after extraction of posterior maxillary teeth. This Pneumatization makes it difficult to place dental implants with appropriate length in the posterior maxillary region. ${ }^{1}$ Sinus lifting and augmentation procedures were introduced, using either indirect close technique or the direct open or lateral approach, with or without simultaneous implant placement. ${ }^{2,3}$

The direct or lateral window technique involves opening a bony window on the lateral surface of the maxilla followed by elevation of the delicate sinus membrane. Unfortunately, perforation of the membrane may be encountered during this procedure at a rate of $10-55 \% ., 5$ Perforation and direct communication to the maxillary sinus lead to scattering of the bone grafting material within the sinus space which may lead to bone graft infection and sinusitis. ${ }^{6,7}$ Actually large membrane perforation may lead to failure of the operation and decreased success rate for implants installed within this augmented sinus. ${ }^{8}{ }^{89}$ It was reported that the majority of sinus perforation occurs from the bur during cutting the lateral window, rather than during the process of membrane separation and elevation..$^{10}$ Ardekian et al. in 2006, reported that $85 \%$ perforation rate of sinus membranes happens when the height of the residual ridge is $3 \mathrm{~mm}$, while the rate is $25 \%$ in residual ridge of $6 \mathrm{~mm} .{ }^{11}$ There are many techniques described to repair these perforations, but they depend on the surgeon's clinical experience and they add to the time and cost of the sinus lift procedure..$^{12,13}$

Piezosurgery was first introduced in the late 80s. Its use depends on ultrasonic microvibration with modulated frequency, from 24.7 to $29.5 \mathrm{KHz}$. The device is capable of performing a selective accurate and controlled cut of the bone without injuring the soft tissues, including blood vessels, nerves, mucosa and the schneiderian membrane. ${ }^{14}$ The high precision level and the high degree of safety needed during oral and maxillofacial operations have encouraged its development. ${ }^{15}$ Among the different surgical operations which are performed safely by the aid of the piezosurgery device, are the autogenous bone graft harvesting, inferior alveolar nerve lateralization, bone splitting, Osteotomy site preparation for implant placement, implant removal and maxillary sinus lifting. ${ }^{16}$ Nowadays, the use of piezosurgery has been increased for sinus membrane elevation. In a study conducted by Wallace et al. in 2007 , they reported only $7 \%$ sinus membrane perforation rate during sinus lift using the piezosurgery device. ${ }^{17}$ Rickert et al. in 2013 , compared the use of piezosurgery tips to the conventional rotating instruments in sinus lift procedure and found no differences in both wound healing and the membrane perforation rate. ${ }^{18}$

Although the feasibility and safety of the use of piezosurgery has been investigated, few prospective clinical studies have been published comparing between the piezosurgery and the conventional turbine. Therefore, the aim of the current study was to evaluate the efficiency and safety of the piezosurgery during direct sinus lifting procedure in comparison with the conventional method.

\section{PATIENTS AND METHODS}

Forty male and female patients, with an age range between 40 to 70 years, who were seeking replacement of one or more missing posterior maxillary teeth with dental implants, were selected for this study. Sample size was calculated using epitools sample calculation website (http://epitools. ausvet.com.au) by entering the means of operation time (20.2 and 18) using the piezoelectric device and the conventional method respectively from a similar study ${ }^{[19]}$, the pooled variance was calculated to be 4.84 , the level of significance was set to be $<0.05$ and the power of the study was assumed to be 0.8 . The generated sample size was 32 patients; 
therefore, taking into consideration the drop out and the attrition of the sample that might occur, a total of 40 patients were selected. Only cases with proper ridge width that is at least $6 \mathrm{~mm}$ and available bone height $3 \mathrm{~mm}$ or less were included in the study. Pregnant females, smokers, patient with infection or pathologic lesion in the maxillary sinus, patients with psychological/mental instability, under radiotherapy, chemotherapy or bisphosphonate medications, or having any uncontrolled systemic disease that might affect the surgery were excluded from the study.

Patients were randomly allocated into one of the two groups; the study group in which the sinus lift procedure was performed by the use of piezosurgical device, and the control group where the operation was done using the conventional rotary instruments. The sample randomization was achieved by the aid of a computer generated randomization table before the start of the surgeries. All work was conducted in accordance with the Declaration of Helsinki 1975, as revised in 2000. A detailed consent was revised and signed by each patient. Before the start of the study an Institutional Review Board (IRB) of Beirut Arab University code was obtained: $2017 \mathrm{H}-0048-$ D-R-0204.
A Cone Beam Computed Tomography (CBCT) (Carestream CS 9000- USA) was requested from each patient to evaluate the anatomy of the sinus and to measure the height and width of the residual ridge from the sinus floor to the alveolar crest (fig. 1). One hour before the surgery patients were given 2g Amoxicillin-clavulinic acid (Augmentin, Glaxosmithkline, England) and 30 minutes before the surgery patients were asked to rinse the mouth for one minute using $0.2 \%$ chlorohexidine mouth wash (Perio-kin, Kin-Spain)

All surgeries were performed by the same operator under complete aseptic conditions. All patients were locally anesthetized using 4\% articaine hydrochloride with 1:100,000 Epinephrine (Septanest, Septodont, France) before the full thickness mucoperiosteal flap was reflected. In the study group, the osteotomy was done using Piezosurgery device (Mectron Dental, Italy) (Fig. 2). The bony cut started with insert OT1 then it was completed using the OT5 insert when becoming close to the membrane.After finishing the osteotomy, the sinus membrane was separated from the bony window using El1 insert then the elevation was completed using inserts EL2 and EL3 (Figs.3 and 4).

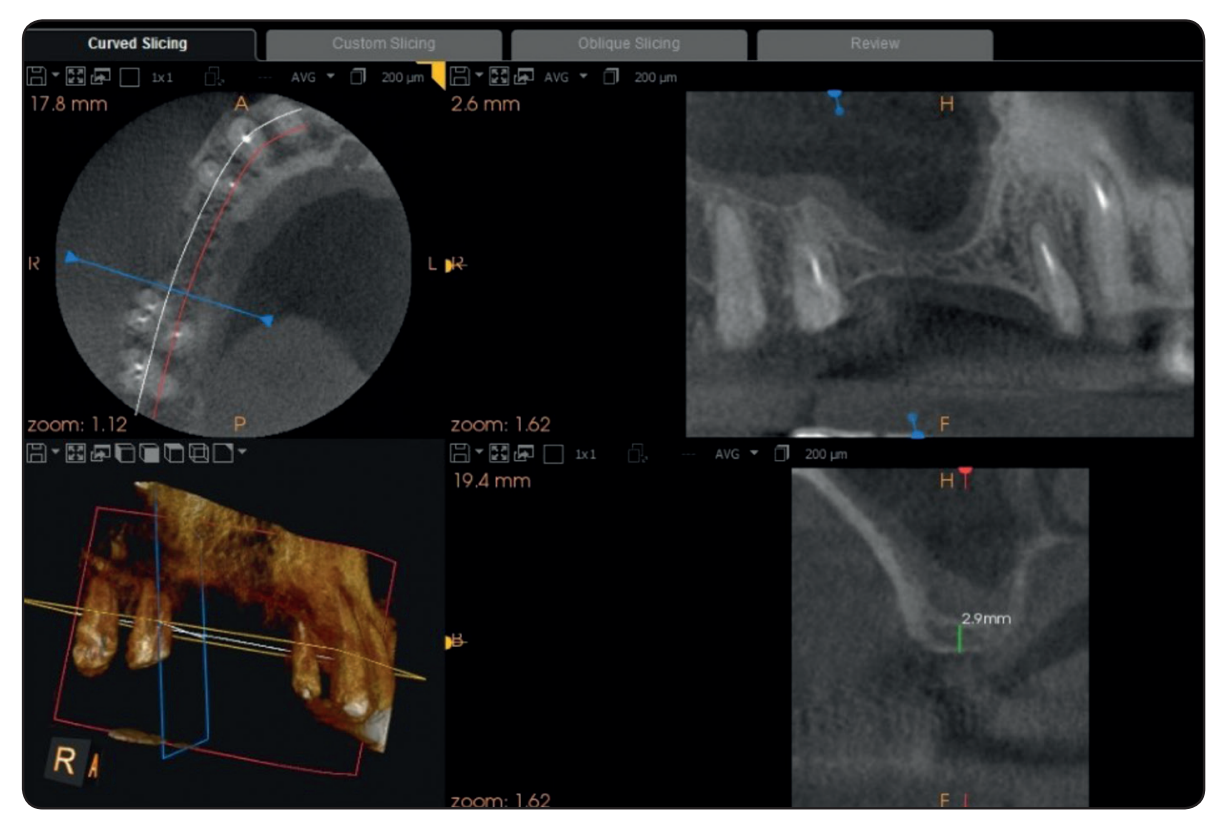

Fig. (1) Preoperative CBCT showing the low bone height in the maxillary posterior area. 


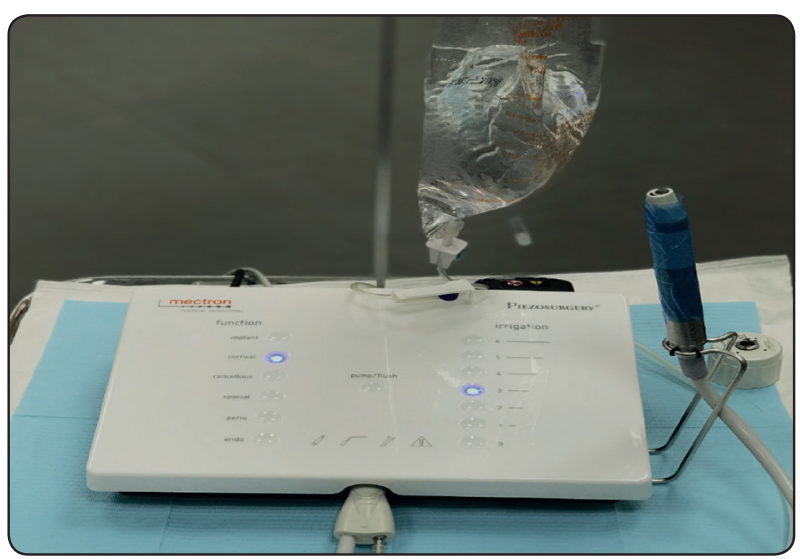

Fig. (2) The piezoelectric device with the handpiece (Mectron Dental, Italy)

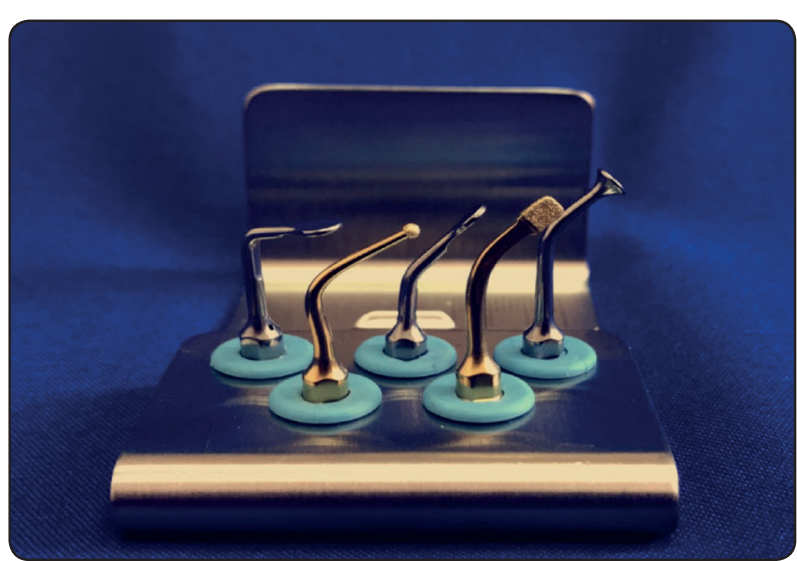

Fig. (3) The Mectron Piezosurgery sinus lift lateral kit Inserts for bone window osteotomy (golden at the front row) and inserts for membrane elevation (silver at the back row).

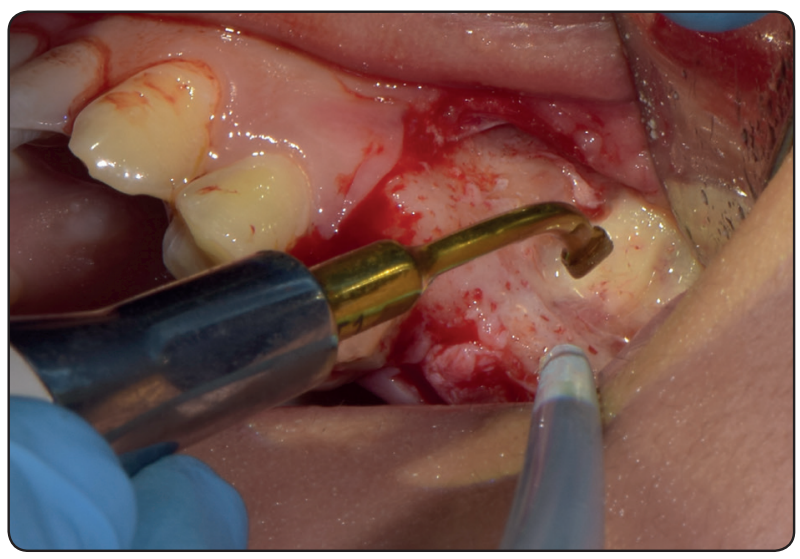

Fig. (4) The typical flap reflection and the Piezosurgery insert (OT1) during cutting the lateral window for sinus lift.
At the control group the bony window was created using a diamond round bur on low speed handpiece, then Schneiderian membrane separation and elevation was performed using the sinus lift elevators (Zimmer Biomet Dental, USA) (Fig.5). In both groups, the lateral residual bone was kept attached to the elevated sinus membrane acting as a new sinus floor. The sinus membrane perforation was recorded after the osteotomy and again when the membrane as elevated. Time of the osteotomy was recoded in minutes starting from the beginning of the osteotomy until the complete elevation of the sinus membrane.

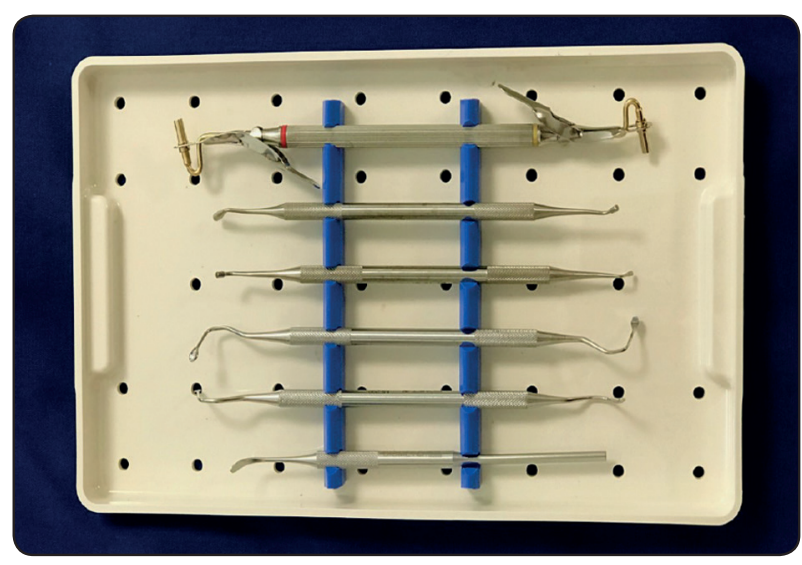

Fig. (5) Lateral sinus lift elevators (Zimmer Biomet Dental, USA).

After proper elevation of the sinus membrane, Xenograft (Cerabone, Botiss Biomaterials $\mathrm{GmbH}$, Germany) was wetted using saline and packed into the sinus. Collagen membrane (Jason membrane, Botiss Biomaterials $\mathrm{GmbH}$, Germany) was adapted to close the sinus bone window and any existing perforations. Flap was closed by interrupted and horizontal mattress sutures using 3/0 silk. After surgery, patients were instructed to take 1g amoxicillin-clavulinic acid (Augmentin, Glaxosmithkline, England) twice daily, $400 \mathrm{mg}$ ibuprofen (Brufen, Abbott laboratories, USA) three times daily, and rinse their moth with chlorhexidine 
0.12\% (Paroex-G.U.M-USA) twice daily for 10 days. Sutures were also removed after 10 days.

Immediate postoperative CBCTs were taken to all cases (Fig.6). Recalls were scheduled on second, seventh and tenth postoperative days. Pain was assessed by the aid of the Visual Analogue Scale (VAS), while the swelling was recorded by a tape-measuring method that takes into account the following measures: the distance between the lateral corner of the eye to the corner of the mouth, intertragic notch to inner canthus of the eye and from the lateral canthus of the eye to the angle of mandible. The mean swelling was then calculated by the sum of the measurements divided by three. Finally, the healing of the operation site was examined for any signs of infection or dehiscence of the flap. The patients were recalled on monthly basis for the following six months in order to detect any arising complications. Implants (Zimmer Biomet 3i, USA) were then inserted six months post-surgically.

Statistical Analysis: Collected data from this study were quantitative and qualitative. The quantitative data were tested for normality using Kolmogorov-Smirnov test. All quantitative variables proved to be not normally distributed except the age. Therefore, they were summarized in form of median and interquartile range while the mean and standard deviation was used for the normally distributed data. The Mann-Whitney $\mathrm{U}$ Test was used as a non-parametric test for the comparison between both groups. For the qualitative data, it was summarized in form percentage using the Fisher exact test for the comparison. The data were analyzed using Statistical Package for the Social Sciences (SPSS version 22).

\section{RESULTS}

This study was carried out on forty patients, of whom 17 (42.5\%) were females and 23 (57.5\%) were males. The age ranged between 43 to 68 years with mean age $55( \pm 7)$ years. All patients completed the 10 days follow up with no attrition of the sample, on the other hand the patients were followed up every month in order to place implants after complete bone formation, four patients dropped out from the treatment at 6 months at the time of implant insertion.

Although the rate of sinus membrane perforation was one patient $(5 \%)$ in the study group and three patients $(15 \%)$ in the control group, there was no statistical significance difference $(P=0.609)$ between groups. The operation time was calculated in minutes starting from the initiation of the osteotomy till the final elevation of the sinus membrane. There was a statistical difference $(P<0.001)$ in time of surgery between the two groups as the median \pm (IQ) time of surgery was $18.64 \pm$ (3.88) in study group which is shorter $14.25 \pm(4.08)$ than control group (Table 1).

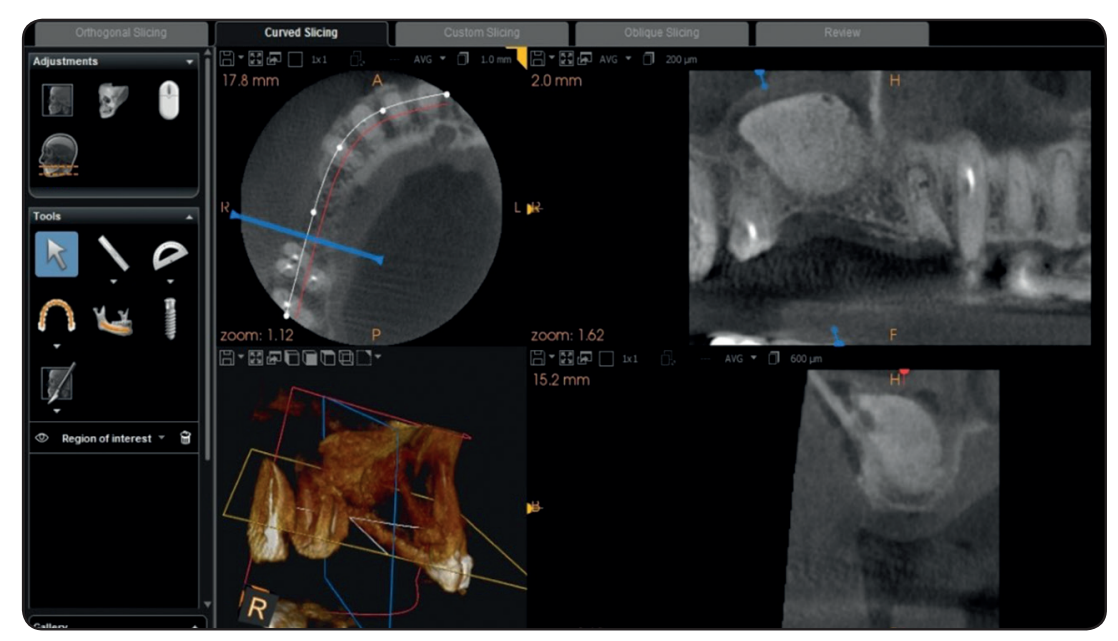

Fig. (6) Immediate Postoperative CBCT showing the augmented sinuscavities using Cerabone graft. 
TABLE (1) comparison of operation time between piezosurgery and conventionaltechnique (in minutes).

\begin{tabular}{|c|c|c|c|}
\hline $\begin{array}{c}\text { Time in } \\
\text { minutes }\end{array}$ & $\begin{array}{c}\text { Piezosurgery } \\
\text { Med/IQ }\end{array}$ & $\begin{array}{c}\text { Conventional } \\
\text { Technique } \\
\text { Min - Max } \\
\text { Med/IQ }\end{array}$ & P Value \\
& $14.9-25.6$ & $12.7-20.5$ & $0.001^{*}$ \\
& $18.64 \pm 3.88$ & $14.25 \pm 4.08$ & \\
\hline
\end{tabular}

*Statistically significant at $p \leq 0.05$

Regarding the healing, all patients showed proper healing with absence of flap dehiscence, pus and infection sings, except one within the control group that showed flap dehiscence on the 2nd postoperative day. For this case, the sutures were removed; refreshment of the wound margin and re-suturing of the flap was performed. The pain perception was assessed by VAS score, where median in study group was $5,2,0$, and in control group was $1.16,3,1$. The pain perception was significantly less in study group on all time points during follow up periods $(\mathrm{P} \leq 0.0001,<0.0001$, and 0.009) (Table 2).

TABLE (2) Comparison of pain sensations between both groups using VAS at different follow up period.

\begin{tabular}{|c|c|c|c|}
\hline $\begin{array}{c}\text { Follow } \\
\text { up }\end{array}$ & $\begin{array}{c}\text { Piezosurgery } \\
\text { Min - Max } \\
\text { Med/IQ }\end{array}$ & $\begin{array}{c}\text { Conventional } \\
\text { Surgery } \\
\text { Min - Max } \\
\text { Med/IQ }\end{array}$ & $P$ Value \\
\hline \multirow{2}{*}{$1^{\text {st }}$ day } & $2-7$ & $4-8$ & \multirow{2}{*}{$0.001 *$} \\
\hline & $5 \pm 1$ & $1.16 \pm 6$ & \\
\hline \multirow{2}{*}{$7^{\text {th }}$ day } & $0-4$ & $1-5$ & \multirow{2}{*}{$0.001 *$} \\
\hline & $2 \pm 1.75$ & $3 \pm 1$ & \\
\hline \multirow{2}{*}{$10^{\text {th }}$ day } & $0-1$ & $0-2$ & \multirow{2}{*}{$0.009 *$} \\
\hline & $0 \pm 0.75$ & $1 \pm 2$ & \\
\hline
\end{tabular}

*Statistically significant at $p \leq 0.05$
Regarding the swelling, the median of study group was 10.66, 10.42 and 10.25, and median of control group was 11.38, 11 and 10.26. Study group showed a significantly lesser post-operative swelling on the 1 st and $7^{\text {th }}$ post-operative days $(\mathrm{P} \leq$ $0.001,<0.001)$ than control group (Table 3 ).

Table (3) Comparison of swelling between both groups at different follow up periods (in centimeters).

\begin{tabular}{|c|c|c|c|}
\hline $\begin{array}{c}\text { Follow } \\
\text { up }\end{array}$ & $\begin{array}{c}\text { Piezosurgery } \\
\text { Min - Max } \\
\text { Med/IQ }\end{array}$ & $\begin{array}{c}\text { Conventional } \\
\text { Surgery } \\
\text { Min - Max } \\
\text { Med/IQ }\end{array}$ & \multirow{2}{*}{ P Value } \\
$\mathbf{1}^{\text {st }} \mathbf{d a y}$ & $10.3-10.8$ & $11.28-13$ & \multirow{2}{*}{$0.001^{*}$} \\
& $10.66 \pm 0.23$ & $11.38 \pm 0.82$ & \\
\cline { 2 - 3 } $\mathbf{7}^{\text {th }}$ day & $10.2-10.56$ & $10.69-11.8$ & \multirow{2}{*}{$0.001^{*}$} \\
& $10.42 \pm 0.21$ & $11 \pm 0.63$ & \\
& & & \multirow{2}{*}{0.108} \\
$\mathbf{1 0}^{\text {th }}$ day & $10.03-10.32$ & $10.1-11.21$ & \\
& $10.25 \pm 0.2$ & $10.26 \pm 0.26$ & \\
\hline
\end{tabular}

*Statistically significant at $p \leq 0.05$

\section{DISCUSSION}

Direct sinus lift procedure had become throughout the years an indispensable surgical technique in order to replace posterior maxillary missing teeth in alveolar ridges with deficient height. Many complications can arise if the procedure was performed without care, such as perforation of the schneiderian membrane, pain, swelling and inappropriate wound healing. The technique of cutting the bony window and the elevation of the membrane are crucial in preventing the aforesaid problems.

The piezosurgery selective cutting of mineralized tissues and preservation of soft tissues makes the device a safe choice in bone removal in delicate sites. Vercellotti et al 2005 stated that by 
using the piezoelectric device, new bone formation is more rapid than with rotating burs when used appropriately. ${ }^{20}$ The aim of this study was to compare the efficiency of piezosurgery versus the conventional technique in direct sinus lift.

Perforation is one of the most common complications during the lateral sinus lift procedure. ${ }^{5}$ In this study, only $5 \%$ of the study group had perforation while the rate was $15 \%$ in the control group, nevertheless the difference was not statistically significant, and this could be attributed that the same experienced oral surgeon performed all surgeries. This is in agreement with Delilbasi et al. $(2013)^{19}$ who compared lateral sinus elevation using both methods and their effect on perforation rate, time of surgery and patient's comfort. On the other hand, Wallace et al (2007) ${ }^{[17]}$ stated that the perforation rate decreases $23 \%$ while using piezosurgery. This percentage is not tested for significance. While Geminiani et al. (2015) ${ }^{21}$ in his retrospective study had found significant difference in perforation rate of the sinus membrane but this was attributed that surgeries were performed by different operators and altered level of experience.

When time of the surgery was recorded from the start of the osteotomy till the complete sinus elevation, the control group was significantly faster than the study group. Many authors compared time between both techniques in different surgical procedures, and most of them found that piezosurgery requires more time for completion of surgeries, ${ }^{22-25}$ except Delilbasi et al. (2013) ${ }^{19}$ found parallel results in their comparison.

Similar to our findings, Atieh et al. (2015) ${ }^{[26]}$, compared both methods of bone removal in lateral sinus augmentation in a meta-analysis, four studies were included in their review. Their results concluded that the studied technique had more prolonged surgical time. In contrary the authors found that the rate of perforation was comparable between both groups, this can be justified that the meta-analysis had a larger sample size.
Also, pain score was evaluated and compared between both groups. Patients in study group experienced significantly lesser pain than the control group. This is in agreement with Piersanti et al (2014) ${ }^{22}$, Mantovani et al. (2014) ${ }^{23}$, and Arakji et al. $(2016)^{24}$ in which the authors compared the pain score between the piezosurgery and the conventional technique in removing mandibular third molars. Lastly, when assessing swelling between both groups, it was significantly more prominent in the control group than the study group. These findings are in agreement with Koçak et al. $(2017)^{27}$, in their study they compared osteotomy between conventional technique and piezosurgery in regards to rhinoplasty. Piersanti et al. (2014) ${ }^{22}$ showed similar findings in regards to swelling while assessing the piezosurgery in removing impacted lower third molars.

\section{CONCLUSION}

Although it seems that piezosurgery is a time consuming device, it is effective in performing a safe sinus membrane elevation as it showed a lower risk for membrane perforation, enhanced postsurgical patient comfort and improved patient's quality of life.

\section{REFERENCES}

1. Ali SA, Karthigeyan S, Deivanai M, Kumar A. Implant rehabilitation for atrophic maxilla: a review. The Journal of Indian Prosthodontic Society. 2014 Sep 1;14(3):196-207.

2. Pal US, Sharma NK, Singh RK, Mahammad S, Mehrotra D, Singh N, Mandhyan D. Direct vs. indirect sinus lift procedure: A comparison. National journal of maxillofacial surgery. 2012 Jan 1;3(1):31.

3. Gonzalez S, Tuan MC, Ahn KM, Nowzari H. Crestal approach for maxillary sinus augmentation in patients with $\leq$ $4 \mathrm{~mm}$ of residual alveolar bone. Clinical implant dentistry and related research. 2014 Dec 1;16(6):827-35.

4. Hernández-Alfaro F, Torradeflot MM, Marti C. Prevalence and management of Schneiderian membrane perforations during sinus-lift procedures. Clinical oral implants research. 2008 Jan 1;19(1):91-8. 
5. Schwartz-Arad D, Herzberg R, Dolev E. The prevalence of surgical complications of the sinus graft procedure and their impact on implant survival. Journal of periodontology. 2004 Apr 1;75(4):511-6.

6. Barone A, Santini S, Sbordone L, Crespi R, Covani U. A clinical study of the outcomes and complications associated with maxillary sinus augmentation Int J Oral Maxillofac Implants. 2006 Jan-Feb;21(1):81-5.

7. Nolan PJ, Freeman K, Kraut RA. Correlation between Schneiderian membrane perforation and sinus lift graft outcome: a retrospective evaluation of 359 augmented sinus.J Oral Maxillofac Surg. 2014 Jan;72(1):47-52.

8. Kim GS, Lee JW, Chong JH, Han JJ, Jung S, Kook MS, Park HJ, Ryu SY, Oh HK. Evaluation of clinical outcomes of implants placed into the maxillary sinus with a perforated sinus membrane: a retrospective study. Maxillofacial Plastic and Reconstructive Surgery. 2016 Dec 1;38(1):50.

9. Becker ST, Terheyden H, Steinriede A, Behrens E, Springer I, Wiltfang J. Prospective observation of 41 perforations of the Schneiderian membrane during sinus floor elevation. Clinical oral implants research. 2008 Dec 1;19(12):1285-9.

10. Valassis JM, Fugazzotto PA. A classification system for sinus membrane perforations during augmentation with option to repair. J Periodontol. 1999;70:692.

11. Ardekian L, Oved-Peleg E, Mactei EE, Peled M. The clinical significance of sinus membrane perforation during augmentation of the maxillary sinus. Journal of Oral and Maxillofacial Surgery. 2006 Feb 28;64(2):277-82.

12. Testori T, Wallace SS, Del Fabbro M, Taschieri S, Trisi P, Capelli M, Weinstein RL. Repair of large sinus membrane perforations using stabilized collagen barrier membranes: surgical techniques with histologic and radiographic evidence of success. International Journal of Periodontics \& Restorative Dentistry. 2008 Jan 1;28(1).

13. Proussaefs P, Lozada J, Kim J, Rohrer MD. Repair of the perforated sinus membrane with a resorbable collagen membrane: a human study. International Journal of Oral \& Maxillofacial Implants. 2004 May 1;19(3).

14. Eggers G, Klein J, Blank J, Hassfeld S. Piezosurgery®: an ultrasound device for cutting bone and its use and limitations in maxillofacial surgery. British Journal of oral and maxillofacial surgery. 2004 Oct 31;42(5):451-3.

15. Landes CA, Stübinger S, Rieger J, Williger B, Ha TK, Sader R. Critical evaluation of piezoelectric osteotomy in orthognathic surgery: operative technique, blood loss, time requirement, nerve and vessel integrity. Journal of Oral and Maxillofacial Surgery. 2008 Apr 30;66(4):657-74.

16. Pereira CC, Gealh WC, Meorin-Nogueira L, Garcia-Júnior IR, Okamoto R. Piezosurgery applied to implant dentistry: clinical and biological aspects.J Oral Implantol. 2014 Jul;40 Spec No:401-8.

17. Wallace SS, Mazor Z, Froum SJ, et al. Schneiderian membrane perforation rate during sinus elevation using piezosurgery: Clinical results of 100 consecutive cases. Int J Periodontics Restorative Dent. 2007;27:413-419.

18. Rickert D, Vissink A, Slater JJ, Meijer HJ, Raghoebar GM. Comparison between conventional and piezoelectric surgical tools for maxillary sinus floor elevation. A randomized controlled clinical trial. Clinical implant dentistry and related research. 2013 Apr 1;15(2):297-302.

19. Delilbasi C, Gurler G. Comparison of piezosurgery and conventional rotative instruments in direct sinus lifting. Implant dentistry. 2013 Dec 1;22(6):662-5.

20. Vercellotti T, Nevins ML, Kim DM, Nevins M, Wada K, Schenk RK, Fiorellini JP. Osseous response following resective therapy with piezosurgery. International Journal of Periodontics \& Restorative Dentistry. 2005 Dec 1;25(6).

21. Geminiani A, Weitz DS, Ercoli C, Feng C, Caton JG, Papadimitriou DE. A comparative study of the incidence of Schneiderian membrane perforations during maxillary sinus augmentation with a sonic oscillating handpiece versus a conventional turbine handpiece. Clinical implant dentistry and related research. 2015 Apr 1;17(2):327-34.

22. Piersanti L, Dilorenzo M, Monaco G, Marchetti C. Piezosurgery or conventional rotatory instruments for inferior third molar extractions? Journal of Oral and Maxillofacial Surgery. 2014 Sep 30;72(9):1647-52.

23. Mantovani E, Arduino PG, Schierano G, Ferrero L, Gallesio G, Mozzati M, Russo A, Scully C, Carossa S. A split-mouth randomized clinical trial to evaluate the performance of piezosurgery compared with traditional technique in lower wisdom tooth removal. Journal of Oral and Maxillofacial Surgery. 2014 Oct 31;72(10):1890-7.

24. Arakji H, Shokry M, Aboelsaad N. Comparison of Piezosurgery and Conventional Rotary Instruments for Removal of Impacted Mandibular Third Molars: A Randomized Controlled Clinical and Radiographic Trial. International Journal of Dentistry. 2016 Aug 14;2016. 
25. Goyal M, Marya K, Jhamb A, Chawla S, Sonoo PR, Singh V, Aggarwal A. Comparative evaluation of surgical outcome after removal of impacted mandibular third molars using a Piezotome or a conventional handpiece: a prospective study. British Journal of Oral and Maxillofacial Surgery. 2012 Sep 30;50(6):556-61.

26. Atieh MA, Alsabeeha NH, Tawse-Smith A, Faggion Jr $\mathrm{CM}$, Duncan WJ. Piezoelectric surgery vs rotary instru- ments for lateral maxillary sinus floor elevation: a systematic review and meta-analysis of intra-and postoperative complications. International Journal of Oral \& Maxillofacial Implants. 2015 Nov 1;30(6).

27. Koçak İ, Doğan R, Gökler O. A comparison of piezosurgery with conventional techniques for internal osteotomy. European Archives of Oto-Rhino-Laryngology. 2017 Jun 1;274(6):2483-91. 